\title{
Multi-Scale Study of the Static Structure of Liquid $\mathrm{Li}_{70}-\mathrm{Bi}_{30}$ Alloy
}

\author{
J.-F. Wax ${ }^{1}$, M.R. Johnson ${ }^{2}$, L.E. Bove ${ }^{3}$, and M. Mihalkovic ${ }^{4}$ \\ ${ }^{1}$ Laboratoire de Physique des Milieux Denses, Université Paul Verlaine - Metz, 57078 Metz Cedex 3, France ${ }^{\mathrm{a}}$ \\ ${ }^{2}$ Institut Laue Langevin, 38042 Grenoble Cedex 9, France \\ ${ }^{3}$ Physique des Milieux Denses, IMPMC, CNRS-UMR7590, Université Pierre et Marie Curie, 75252 Paris, France \\ ${ }^{4}$ Institute of Physics, Slovak Academy of Sciences, 84511 Bratislava, Slovakia
}

\begin{abstract}
A multi-scale approach is applied to study the static structure of $\mathrm{Li}_{70}-\mathrm{Bi}_{30}$ liquid alloy. In order to describe the interatomic interactions in this non-simple metal, we make a set of three empirical pair potentials fit $a b$-initio computations of the forces and energy in this system. Large scale classical simulations are performed using these fitted potentials from which the partial and total structures are computed. These results are validated by comparison with new experimental neutron data. The partial structure clearly indicates heterocoordination, which is consistent with a marked ionic nature of the bonding, in agreement with earlier electrical resistivity measurements.
\end{abstract}

\section{Introduction}

Molecular dynamics (MD) simulations have demonstrated how helpful they can be for the study of alloys, leading easily to the knowledge of partial structures and chemical order [1, 2]. However, non simple metals and alloys are difficult to describe using pair potentials.

Although ab-initio methods are accurate, they are limited as far as the number of particles is concerned. Thus, multi-scale approaches, modelling the interactions from $a b$-initio computations and using these models on a larger space-scale (namely in classical MD) are required.

In this study, we apply a method developed by Mihalkovic et al. [3] to the case of liquid $\mathrm{Li}_{70}-\mathrm{Bi}_{30}$ alloy, a composition close to the $\mathrm{Li}_{3} \mathrm{Bi}$ compound forming one. The temperature is $1073 \mathrm{~K}$ and the corresponding density, $\rho=4.73 \mathrm{~g} / \mathrm{cm}^{3}$. Under these conditions, this alloy is far from being a simple metal (i.e. nearly-free electronlike) as shown by its electrical resistivity [4]. Indeed, it is suspected to lie on the borderline between a metallic and an ionic liquid.

In order to illustrate the interest of this method, which we will shortly describe in section 2 , we will focus on the

\footnotetext{
${ }^{a}$ Corresponding author: wax@univ-metz.fr
}

static structure of this alloy. The corresponding results will be displayed and discussed in section 3 .

\section{Multi-scale approach}

\subsection{Description}

In a first step, ab-initio MD simulations are performed which provide us with configurations from which energies and forces are obtained; these are considered as reference data. We used VASP code and applied it to a box filled with 200 particles over a 30 ps simulated time, under the desired thermodynamic conditions.

In a second step, an analytical expression

$u_{i j}(r)=\frac{C_{i j}^{(1)}}{r \eta_{i j}^{(1)}}+\frac{C_{i j}^{(2)}}{r \eta_{i j}^{(2)}} \cos \left(k_{i j} r+\Phi_{i j}\right)$

is fitted in order to reproduce the ab-initio energies and forces. The parameter values thus obtained are displayed in Table 1.

Then, in a final step, these fitted potentials are used to perform classical MD simulations. We considered 13500 particles over $10 \mathrm{ps}$ in order to obtain the structural functions of interest. From the partial pair distribution 
functions, $g_{i j}(r)$, Ashcroft-Langreth and Bathia-Thornton partial structure factors were computed and then the total one for neutrons (see Refs. 1 and 2 for more details).

Nevertheless, some checks are necessary and the fitting procedure can be tested by comparing ab-initio and classical MD predictions for the partial structures. Besides, the realism of the model of interactions can be ascertained by comparing simulation and experimental results.

Table 1. Fitted parameters for the 3 pair potentials; units are such that energies are in $\mathrm{eV}$ if distances are in Angströms.

\begin{tabular}{|c|c|c|c|}
\hline & Li-Li & Li-Bi & Bi-Bi \\
\hline $\mathrm{C}_{\mathrm{ij}}{ }^{(1)}$ & 396.027 & 2612.40 & 2080.54 \\
\hline$\eta_{\mathrm{ij}}{ }^{(1)}$ & 8.7357 & 12.4689 & 7.2847 \\
\hline $\mathrm{C}_{\mathrm{ij}}{ }^{(2)}$ & 0 & -55.123 & -58.802 \\
\hline$\eta_{\mathrm{ij}}{ }^{(2)}$ & 0 & 5.4404 & 4.6707 \\
\hline $\mathrm{k}_{\mathrm{ij}}$ & 0 & 2.2036 & 2.5329 \\
\hline$\phi_{\mathrm{ij}}$ & 0 & 5.5387 & 5.2602 \\
\hline
\end{tabular}

\subsection{Pair potentials}

As can be observed from the data in Table $1, u_{L i L i}(r)$ is purely repulsive: this feature is consistent with ionic-like behaviour. Moreover, considering the curves of these potentials (Fig. 1), Bi-Bi core-repulsion is wider than both others while Li-Bi interaction is strongly attractive: all these features are promoting hetero coordination tendencies.

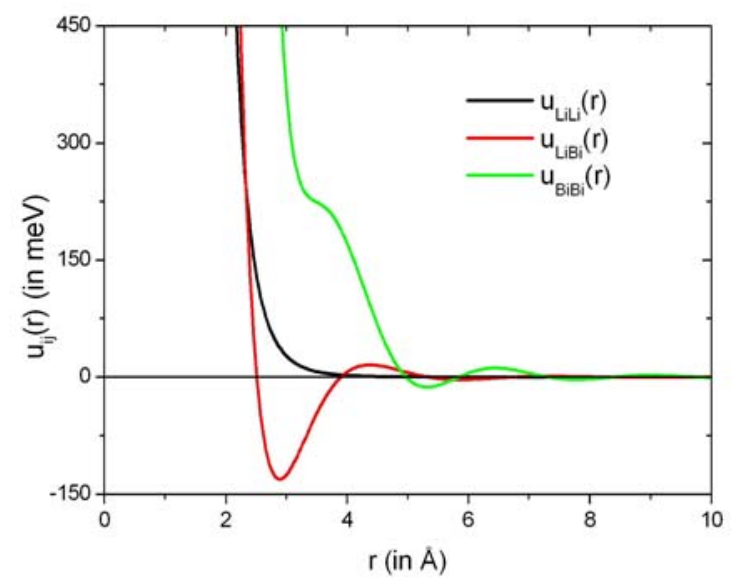

Fig. 1. Effective pair potentials fitted to ab-initio simulations.

\subsection{Partial pair distribution functions}

This hetero coordination is clearly displayed considering the partial pair distribution functions (Fig. 2). Indeed, $g_{L i B i}(r)$ has the highest first peak, indicating that Li atoms are mostly surrounded by $\mathrm{Bi}$ atoms, and vice-versa. Besides, considering the particular shape of $g_{B i B i}(r)$, its very low first peak indicates that the first neighbour of a $\mathrm{Bi}$ atom is rarely of the same kind and that most of the $\mathrm{Bi}$ atoms are pushed back into the second neighbour shell. Thus, this is consistent with the existence of loosely bound $\mathrm{Li}_{3}-\mathrm{Bi}$ compounds and with the increase of the ionic character of the bonding $[4,5]$.

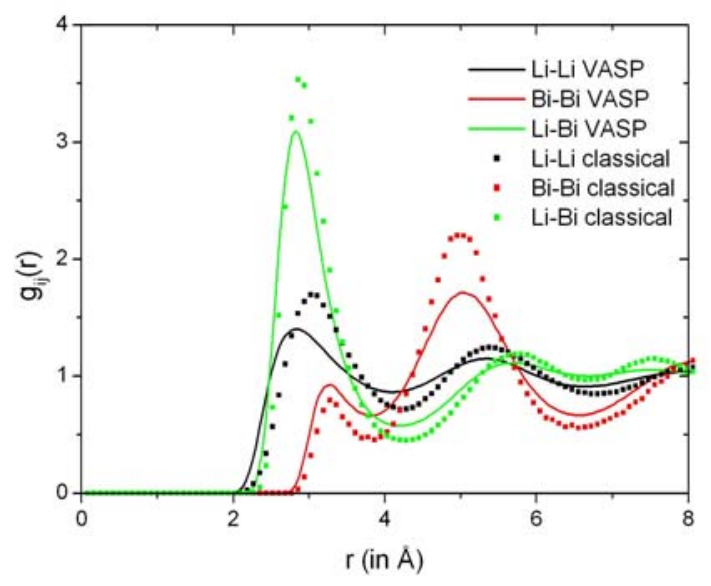

Fig. 2. Partial pair distribution functions obtained from $a b$-initio (VASP) and classical simulations.

Comparing now classical and ab-initio MD results, even if there is not a perfect quantitative agreement, the main features are recovered, namely (i) the peak positions, (ii) the hierarchy between peak heights, and even (iii) the peculiar first peak of $g_{B i B i}(r)$. Therefore, we can consider that the fitted pair potentials correctly reproduce the interactions from $a b$-initio $\mathrm{MD}$.

\subsection{Neutron structure factor}

In order to check the realism of the fitted potentials, it is necessary to consider experimental measurements. Therefore, we examine now the total structure factor as measured in neutron diffusion experiments. These data were obtained by integrating over energy the dynamic structure factor, $S(q, \omega)$, as obtained from inelastic scattering experiments [6]. These were performed at ILL's hot source. We thus obtained $\mathrm{S}(\mathrm{q})$ for several scattering vectors between 0.5 and $2 \AA^{-1}$. They are displayed in figure 3 with the results that we obtained by combining the partial pair structure factors using the appropriate diffusion lengths.

As can be seen, the agreement is excellent, which indicates that our fitted potentials describe the interactions in a realistic way.

\section{Structure of the alloy}




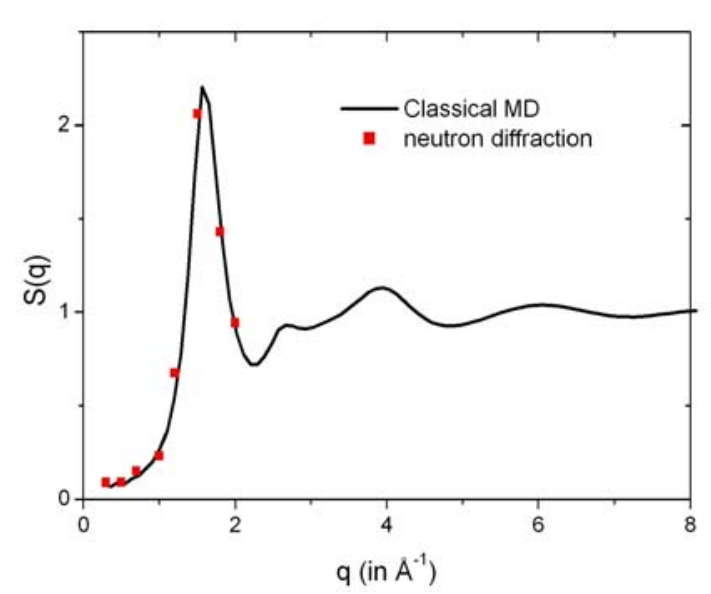

Fig. 3. Total neutron structure factor.

\subsection{Bhatia-Thornton structure factors}

From the partial structure factors, we can also easily compute the so-called Bathia-Thornton structure factors (Fig. 4). These are useful to consider topological and chemical orders. It can be seen that $S_{N N}(q)$ is rather common, demonstrating that there is no particular topological order.

On the other hand, $S_{C C}(q)$ is remarkable. Its low- $q$ limit $S_{C C}(0) \approx 0.011$ which is really less than 0.21 , the value for an ideal mixture of same composition: this clearly demonstrates strong hetero coordination tendencies which could lead to compound formation. It also exhibits a marked peak at about half the position of the main peak of $S_{N N}(q)$. This indicates composition anomalies on a space-range equal to about twice the interatomic distance. All these features are consistent with the existence of loosely bound $\mathrm{Li}_{3} \mathrm{Bi}$ compounds.

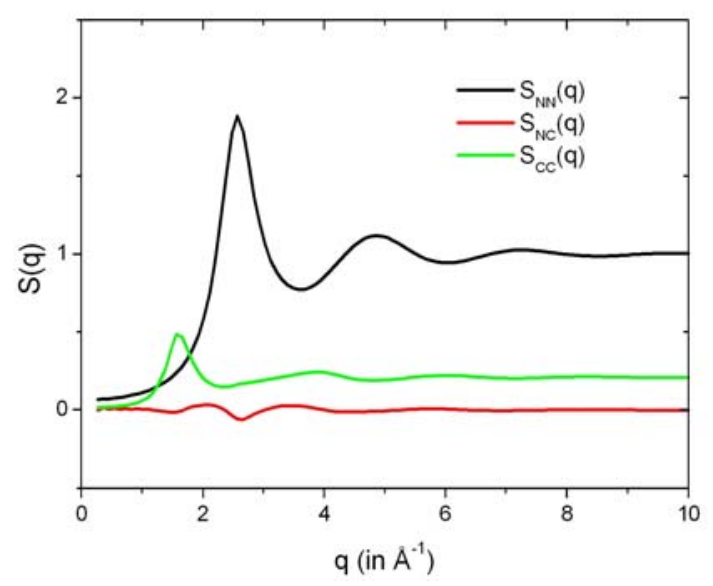

Fig. 4. Bhatia Thornton partial structure factors obtained from classical MD.

This interpretation is corroborated by the phase diagram [7] which displays an intermetallic $\mathrm{Li}_{3} \mathrm{Bi}$ compound. Electrical resistivity measurements [8] also show a sharply peaked dependence with composition: while it is $25 \mu \Omega$.cm for pure $\mathrm{Li}$ and $130 \mu \Omega$.cm for pure $\mathrm{Bi}$, it reaches about $2000 \mu \Omega$.cm at the $\mathrm{Li}_{3} \mathrm{Bi}$ composition.

\section{Conclusion}

We applied a multi-scale approach developed by Mihalkovic et al. to study the static structure of liquid $\mathrm{Li}_{70}-\mathrm{Bi}_{30}$ alloy. This is far from a simple metal (inter metallic compound in the phase diagram, peaked dependence of the resistivity versus composition) and is thus very difficult to describe. Indeed, it is not freeelectron like and usual methods are unable to predict the effective pair interactions.

Nevertheless, Mihalkovic et al. approach succeeds in predicting the structure qualitatively and even quantitatively. Our computations agree remarkably well with neutron experiments. They confirm the structural particularities of the mixture, namely a strong hetero coordination and a peculiar partial Bi-Bi structure. These observations are consistent with the persistence of loosely bound $\mathrm{Li}_{3} \mathrm{Bi}$ compounds into the liquid state.

In order to refine our understanding of this alloy, as well as to confirm this hypothesis, an extension of this study to other compositions, as well as to dynamic properties such as diffusion and collective excitations is in progress.

\section{References}

1. J.-F. Wax and N. Jakse, Physical Review B 75, 024204 (2007)

2. J.-F. Wax, Physica B 403, 4241 (2008)

3. M. Mihalkovic, C.L. Henley, M. Widom, P. Ganesh, arXiv:0802.2926v2 [cond-mat.mtrl-sci]

4. P. Heitjans, G. Kiese, C. van der Marel, H. Ackermann, B. Bader, P. Freiländer, H.J. Stöckmann, Hyperfine Interactions, 15-16, 569 (1983)

5. K. Hackstein, S. Sotier, E. Lüscher, Journal de Physique (Colloques) C8, 49 (1980)

6. L.E. Bove, to be published

7. J. Sangster, A.D. Pelton, Journal of Phase Equilibria 12, 447 (1991)

8. G. Steinleitner, W. Freyland, F. Hensel, Berichte der Bunsengesellshaft 79, 1186 (1975)

\section{Acknowledgments}

The PMMS (Pôle Messin de Modélisation et de Simulation) is greatly acknowledged for providing us with computer time.

M.Mihalkovic was supported by grant VEGA 2/0157/08. 